\title{
Staged surgery for advanced cardiac intimal sarcoma involving the right atrium and the inferior vena cava
}

\author{
Yu-An Chen ${ }^{1}$, Yueh Li $^{2}$, Jen-chieh Lee ${ }^{1}$, and JENG-WEI CHEN ${ }^{1}$ \\ ${ }^{1}$ National Taiwan University Hospital \\ ${ }^{2}$ Far Eastern Memorial Hospital
}

June 24, 2021

\begin{abstract}
Intimal sarcomas simultaneously involving the right atrium and the inferior vena cava are rare. We report an advanced cardiac intimal sarcoma in the right atrium of a 19-year-old man that was complicated by tumor-related inferior vena cava thrombosis. We initially performed partial tumor resection and vena cava thrombectomy to resolve the circulatory obstruction, because complete resection was difficult due to the invading malignancy and an unclear margin. The patient received adjuvant chemoand radiotherapy along with anticoagulant therapy. After 3 months, the border of the residual sarcoma was clear, and the patient underwent a secondary complete sarcoma excision (including that of the right atrium) and a suprahepatic vena cava reconstruction. At the 2-year follow-up, there was no tumor recurrence. We conclude that aggressive treatment and a staged complete resection can lead to improved outcomes for advanced cardiac intimal sarcoma with poor prognosis.
\end{abstract}

\section{Hosted file}

JCS_Primary_Cardiac_Intimal_Sarcoma.doc available at https://authorea.com/users/421691/ articles/527615-staged-surgery-for-advanced-cardiac-intimal-sarcoma-involving-the-rightatrium-and-the-inferior-vena-cava 